\title{
Prospective Analysis of Patients Treated with Chemotherapy in Metastatic Adenocarcinoma of Lung-Single Centre Experience
}

\author{
B. J. Srinivasa*, L. P. Bhanu, Girish Badarke, Mohammad Nasiruddin, Sulav Sapkota, \\ D. Tousif, Vishal Kulkarni, P. K. Kiran, Vinu Sarathy, G. S. Deepika, Amritanshu Ram, \\ Raghavendra Rao, Shekar Patil, Radheshyam Naik
}

Department of Medical Oncology, HCG SC, Bangalore, India

Email: *sripav77@yahoo.co.in

How to cite this paper: Srinivasa, B.J., Bhanu, L.P., Badarke, G., Nasiruddin, M., Sapkota, S., Tousif, D., Kulkarni, V., Kiran, P.K., Sarathy, V., Deepika, G.S., Ram, A., Rao, R., Patil, S. and Naik, R. (2017) Prospective Analysis of Patients Treated with Chemotherapy in Metastatic Adenocarcinoma of Lung-Single Centre Experience. Journal of Cancer Therapy, 8, 838-844. https://doi.org/10.4236/jct.2017.89073

Received: August 14, 2017

Accepted: September 17, 2017

Published: September 20, 2017

Copyright $\odot 2017$ by authors and Scientific Research Publishing Inc. This work is licensed under the Creative Commons Attribution International License (CC BY 4.0).

http://creativecommons.org/licenses/by/4.0/ (c) (i) Open Access

\begin{abstract}
Background: Lung cancer is the leading cause of cancer-related death worldwide with an estimated 1.6 million new cases diagnosed each year. According to standard guidelines in NSCLC, the effectiveness of combined chemotherapy regimen comprises of pemetrexed and platinum, and very few reports are available in Indian population. This study shows the experience with pemetrexed and platinum combination chemotherapy in metastatic NSCLC in first line setting. Methods: Total 61 patients with following inclusion criteria were recruited such as: 1) PS of 1 and 2; 2) NSCLC of non-squamous histology; 3) locally advanced or metastatic disease at diagnosis; 4) should have received minimum 3 cycles of pemetrexed and platinum as first line treatment and or continued for 6 cycles; 5) EGFR/ALK mutated or EGFR/ALK unknown patients but must have received upfront chemo. Primary endpoint of the present study is to assess Disease Control Rate (DCR) (CR + PR + SD). Progression Free Survival, OS and Toxicity assessment were secondary endpoints. Results: The mean number of average chemotherapy cycles was found to be 4.38 . The range of chemotherapy was $2-6$ cycles. Disease control rate, defined as (CR + PR + SD), was seen in $44(72.1 \%)$ patients. (PR in $26(42.62 \%)$, SD 18 (29.5\%)), Progressive disease (PD) was observed in 17 (27.9\%) patients. Overall study showed that patients had PFS of 9.414 months (95\% CI $6.709-12.120$ ) and OS of 13.437 months (95\% CI 10.721 - 16.153). Conclusion: Pemetrexed and platinum combination is effective and well-tolerated chemotherapy regimen in patients with metastatic adenocarcinoma lung cancer patients. Pemetrexed and cisplatin may be more effective in particular. Early switch over to TKIs is preferred immediately after obtaining molecular subtype result.
\end{abstract}




\section{Keywords}

Pemetrexed, Carboplatin, NSCLC, Cisplatin, EGFR

\section{Introduction}

Lung cancer is the leading cause of cancer-related death worldwide with an estimated 1.6 million new cases diagnosed each year [1]. Non-small cell lung cancer (NSCLC) accounts for $80 \%$ of all cases of lung cancer, and is further divided into several subgroups as adenocarcinoma, squamous cell carcinoma, large cell carcinoma, and others with $65 \%$ to $75 \%$ of them having locally advanced or metastatic disease [2]. The prognosis for advanced-stage disease remains poor, with a median overall survival (OS) of approximately a year on recent clinical trials. According to the American Society of Clinical Oncology and National Comprehensive Cancer Network (NCCN) guidelines, patients with a performance status of 0 or 1 have to be treated with a combination of 2 cytotoxic drugs in first-line therapy [3]. In patients with metastatic NSCLC of non-squamous histology, current guidelines recommend the combination of cisplatin and pemetrexed as first line induction treatment [1].

Pemetrexed is a multitargeted antifolate cytotoxic chemotherapy agent, which inhibits at least three target enzymes in the folate pathway (thymidylate synthase, dihydrofolate reductase, and glycinamide ribonucleotide formyl transferase). Consequently, pemetrexed interferes with the synthesis of both pyrimidine and purine, thereby effectively inhibiting both DNA and RNA synthesis [5]. Pemetrexed was approved in second line therapy in NSCLC based on a phase III trial comparing pemetrexed versus docetaxel. In this trial, pemetrexed showed a similar clinical activity and a lower rate of myelosuppression compared to docetaxel [4] [5]. In a secondary analysis, pemetrexed was demonstrated to be more active in non-squamous cell tumours, leading to its restriction to patients with non-squamous histology [6].

Pemetrexed and cisplatin has become the standard first line treatment regimen in metastatic NSCLC, after the first prospective analysis by Scagliotti et al. [7]. It showed that, patients treated with pemetrexed and cisplatin combination in advanced NSCLC, has overall survival benefit and better tolerability compared to gemcitabine and cisplatin combination. Carboplatin has less response rates but better toxicity profile and is preferred, as most of patients with advanced NSCLC have associated comorbidities. Carboplatin is non-inferior to cisplatin, if strict dose adherence is maintained [8] [9]. However, it may have inferior survival in non-squamous cancers in combination with paclitaxel, docetaxel or gemcitabine [10]. This study reports about the experience of using pemetrexed and platinum combination chemotherapy in metastatic NSCLC in first line setting.

\section{Materials and Methods}

Patients receiving treatment for metastatic NSCLC, followed prospectively, at Health Care Global Enterprises Ltd., Bangalore between March 2014 and December 2015. 
Within the study period, 73 NSCLC patients received pemetrexed and platinum-based combination chemotherapy. Of these, 61 patients fulfilled the following inclusion criteria: 1) PS of 1 and 2; 2) NSCLC of non-squamous histology; 3) locally advanced or metastatic disease at diagnosis; 4) should have received minimum 3 cycles of pemetrexed and platinum as first line treatment and or continued for 6 cycles; 5) EGFR/ALK mutated or EGFR/ALK unknown patients but must have received upfront chemo. Patients with non-adenocarcinoma histology and EGFR/ALK mutation treated with upfront TKI were excluded. Pemetrexed $500 \mathrm{mg} / \mathrm{m}^{2}$, Carboplatin (AUC5), or Cisplatin $75 \mathrm{mg} / \mathrm{m}^{2}$ given once in 3 weeks. Dose adjustments made as per standard guidelines. Primary endpoint of the study was to Assess of Disease control rate (DCR - CR + PR + SD). Progression Free survival (PFS), Overall Survival (OS) and Toxicity assessment were secondary endpoints.

\section{Statistical Analysis}

PFS measured from the start date of the first pemetrexed and platinum cycle. Survival analysis performed using the Kaplain-Meier method. Spearman's rank correlation used to evaluate the relationship between performance status and number of treatment cycles.

\section{Results}

Of the 61 patients, receiving chemotherapy and platinum analogues, pemetrexed and carboplatin were given in $45(73.77 \%)$ cases while pemetrexed and cisplatin was administered to $16(26.23 \%)$ cases. The mean number of average chemotherapy cycles was 4.38 . The range of chemotherapy was $2-6$ cycles.

Following chemotherapy treatment, Disease control rate, defined as (CR + PR + SD), seen in 44 (72.1\%) patients. (PR in 26 (42.62\%), SD 18 (29.5\%)). Progressive disease (PD) was observed in 17 (27.9\%) patients. (Figure 1)

Responses in the molecular subtypes were as follows (Figure 2). The EGFR mutated patient had DCR in $14(31.8 \%)$ patients ((PR in $9(34.6 \%)$, SD 5 $(27.8 \%))$. and PD was seen in $2(11.8 \%)$ patients. The EGFR wild type patient

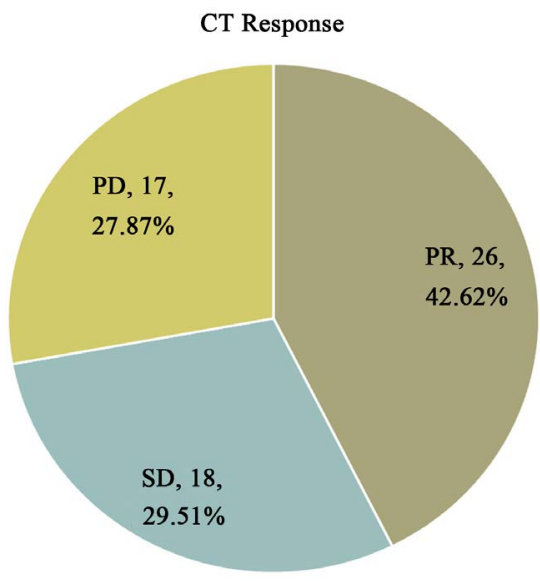

Figure 1. Response rates in study population: PR—Partial Response; SD—Stable Disease; PD_Progressive Disease. 


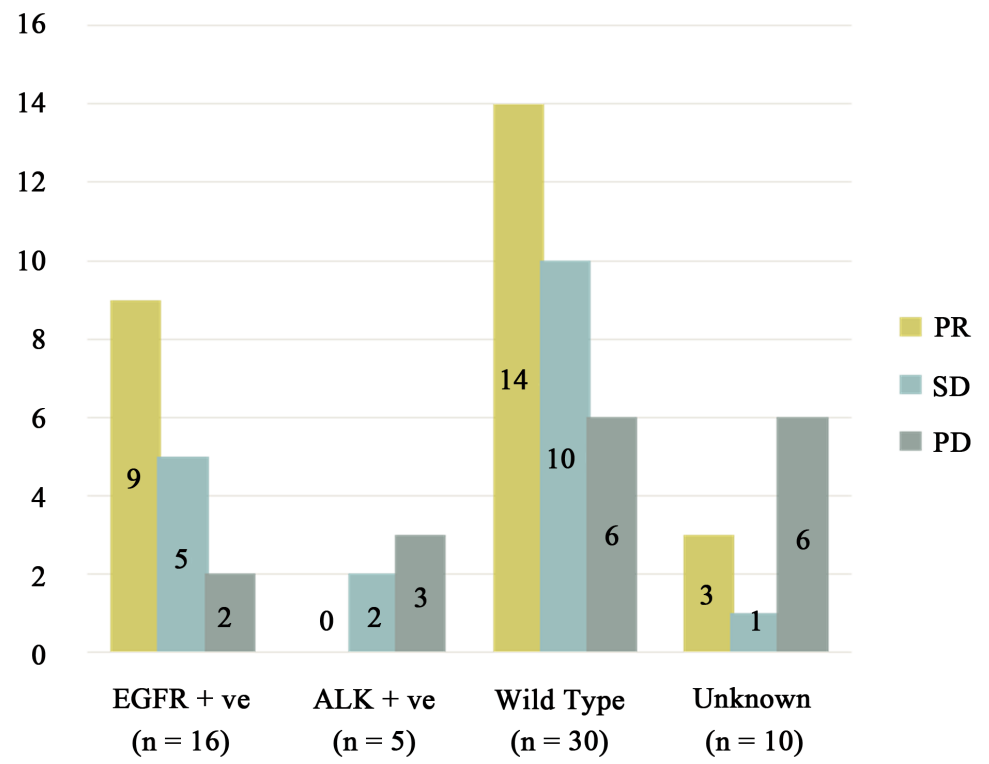

Figure 2. Chemotherapy response in molecular subtypes.

had DCR in 24 (54.5\%) patients (PR in 14 (53.9\%), SD 10 (35.6\%)), PD was seen in 6 (15.87\%). Other molecular subtype ALK mutated DCR in 2 (4.6\%) patients (no PR, SD $2(11.1 \%))$. PD was seen in $3(17.6 \%)$ patients. Unknown molecular status had DCR in 4 (9.1\%) patients (PR in 3 (11.5\%), SD 1 (5.6\%)). PD was seen in $6(35.3 \%)$ patients.

In this study, patients receiving pemetrexed plus carboplatin regimen had DCR of 30 (68.2\%) (PR in 17 (65.38\%), SD in 13 (72.22\%)) and it was 14 (31.8\%) in (PR in $9(34.62 \%)$, SD in $5(27.78 \%)$ ) those receiving pemetrexed plus cisplatin regimen (Figure 3 ).

Overall study patients had PFS (Figure 4) of 9.414 months (95\% CI 6.709 - 12.120) and OS (Figure 5) of 13.437 months (95\% CI 10.721 - 16.153). PFS with pemetrexed and cisplatin regimen was 13 months (95\% CI $7.724-18.501)$ as

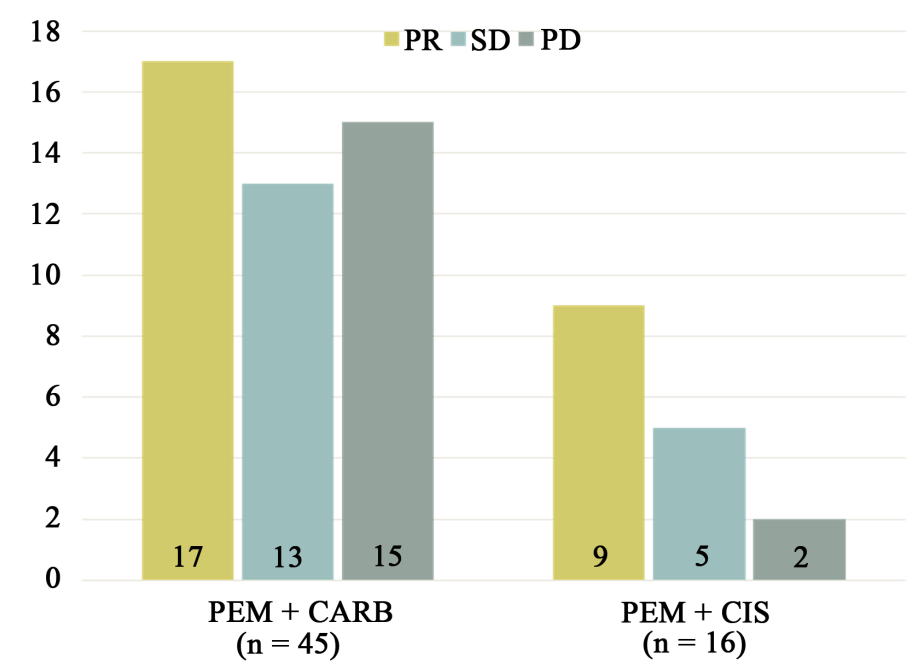

Figure 3. Response with platinum based regimen. 


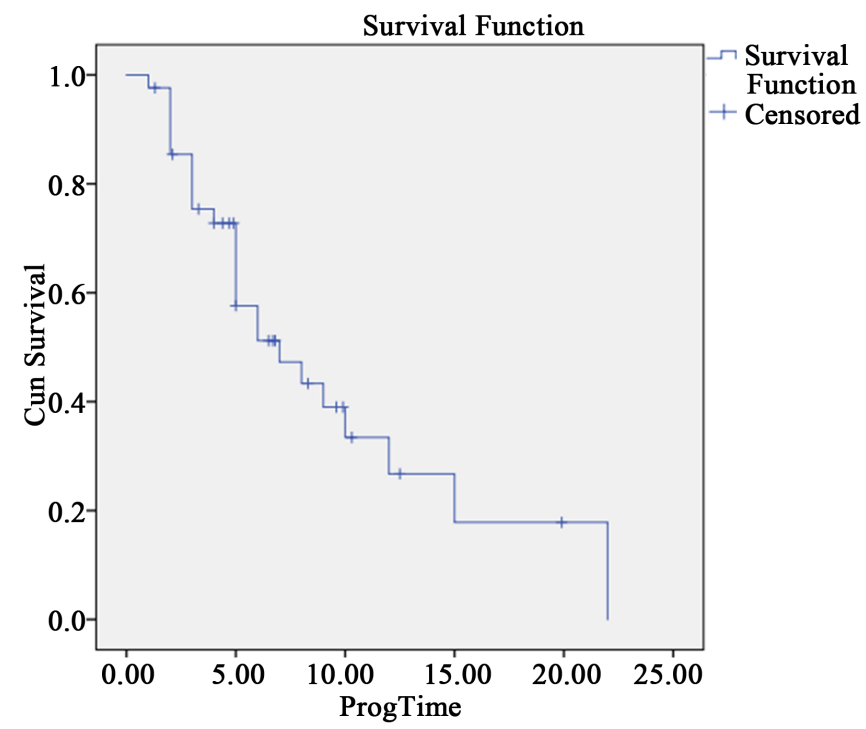

Figure 4. Kaplan-Meier progression free survival of study population.

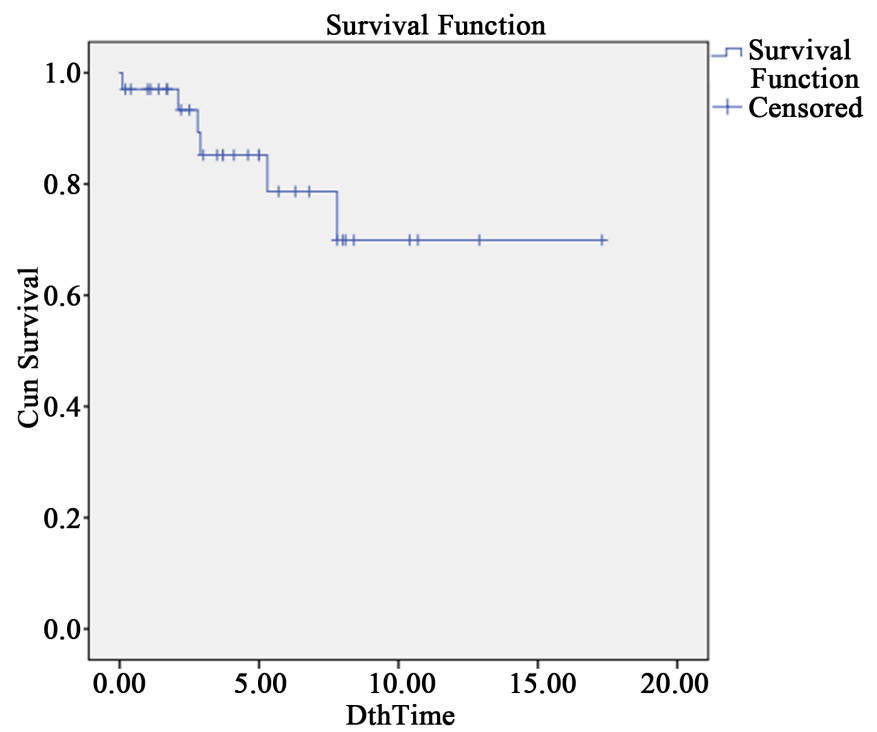

Figure 5. Kaplan-Meier overall survival of study population.

compared to 8 months (95\% CI 5.217 - 10.654) with pemetrexed and carboplatin regimen $(p=0.057)$. A total number $40(65.6 \%)$ patients tolerated chemotherapy well without any side effects. Other side effects as thrombocytopenia was seen in $11(18 \%)$.

\section{Discussion}

Metastatic/Locally advanced NSCLC patients were treated with pemetrexed and platinum combination chemotherapy in first line setting. Most of the patients received average 4 cycles of chemotherapy. DCR (CR + PR + SD), of study population was seen in $44(72.1 \%)$ patients. (PR in $26(42.62 \%)$, SD 18 (29.5\%)), There was no CR. Progressive disease (PD) was observed in 17 (27.9\%) patients. 
Sub type of EGFR mutated patients had lower DCR of 14 (31.8\%) patients compared to EGFR wild type patients having DCR of 24 (54.5\%). Overall study patients had PFS of 9.414 months and OS of 13.437 months.

Phase II study with pemetrexed and carboplatin reported PR in 12 (24\%) patients and SD in 25 (50\%) patients [11]. Significantly better responses observed in this study, even with the lesser dose of carboplatin (AUC5 vs. AUC 6). Phase III study reported objective response rate of $30 \%$ with pemetrexed and cisplatin [7], which is comparable to DCR of $31.8 \%$ in this study, though number of patients, were less on this regimen. As this study included EGFR mutated patients taking chemotherapy upfront also for analysis, other studies may not be comparable.

Meta-analysis by Ardizzoni A. et al. [9] has shown that cisplatin is superior to carboplatin. In accordance with the same, this study results have shown lesser response with cisplatin (31.8\% vs. $68.2 \%)$ but higher PFS than carboplatin (13 months vs. 8 months) which was statistically significant.

Gronberg B.H. et al. [8] reported OS of 7.3 months compared to 13 months in this study on pemetrexed and carboplatin regimen. Survival may be more, because this study had biologically favourable EGFR mutated patients. Similarly, Scagliotti G.V. et al. [7] reported OS of 10 months, which is higher compared to 8 months in this study on pemetrexed and cisplatin regimen.

We had molecular subtype EGFR+ (16 patients) and ALK+ (5 patients). These patients continued on chemotherapy in view of continued clinical improvement after $1^{\text {st }}$ cycle and planned to start on TKI after 4 cycles of chemotherapy. Sequist L.V. et al. [12] reported around $23 \%$ to $44 \%$ response rates and PFS of 6.3 months in patients with EGFR mutated patients in afatinib vs. cisplatin and pemetrexed chemotherapy trial. Same subtype of patients in this study had response rate of $31.8 \%$, which is comparable. PFS and OS was higher (around 10 months) than the above-mentioned study. This may be the result of all patients continuing on TKIs after 4 cycle of chemotherapy.

Most of the patients $40(65 \%)$ in present study tolerated chemotherapy well with asymptomatic grade 3 to 4 neutropenia and thrombocytopenia observed in around $20 \%$ of patients which is almost similar to other studies $(7,8)$ reported elsewhere.

Limitation of this study is small sample size, maintenance treatment data is not collected and inclusion of biologically favourable molecular subtype.

\section{Conclusion}

Pemetrexed and platinum combination is effective and well-tolerated chemotherapy regimen in patients with metastatic adenocarcinoma lung cancer patients. Pemetrexed and cisplatin may be more effective in particular. Early switch over to TKIs is preferred immediately after obtaining molecular subtype result.

\section{Conflict of Interest}

The authors declare that they have no conflict of interest. 


\section{References}

[1] Kim, D.H., Kinmond, A., Gilani, S., et al. (2015) Efficacy and Toxicity of Maintenance Pemetrexed Following Induction Treatment with Pemetrexed plus Cisplatin for Advanced Non-small Cell Non-squamous Carcinoma of the Lung. Forum of Clinical Oncology, 6, 14-18. https://doi.org/10.1515/fco-2015-0008

[2] Zhang, G.Z., Jiao, S.C. and Meng, Z.T. (2010) Pemetrexed plus Cisplatin/Carboplatin in Previously Treated Locally Advanced or Metastatic Non-Small Cell Lung Cancer Patients. Journal of Experimental \& Clinical Cancer Research, 29, 38. https://doi.org/10.1186/1756-9966-29-38

[3] Stinchcombe, T.E., Borghaei, H., Barker, S.S., et al. (2016) Pemetrexed with Platinum Combination as a Backbone for Targeted Therapy in Non-Small Cell Lung Cancer. Clinical Lung Cancer, 17, 1-9.

[4] Hanna, N., Shepherd, F.A., Fossella, F.V., et al. (2004) Randomized Phase III Trial of Pemetrexed versus Docetaxel in Patients with Non-Small-Cell Lung Cancer Previously Treated with Chemotherapy. Journal of Clinical Oncology, 22, 1589-1597. https://doi.org/10.1200/JCO.2004.08.163

[5] Cohen, M.H., Johnson, J.R., Wang, Y.C., et al. (2005) FDA Drug Approval Summary: Pemetrexed for Injection (Alimta) for the Treatment of Non-Small Cell Lung Cancer. Oncologist, 10, 363-368. https://doi.org/10.1634/theoncologist.10-6-363

[6] Tomasini, P., Barlesi, F., Mascaux, C., et al. (2016) Pemetrexed for Advanced Stage Nonsquamous Non-Small Cell Lung Cancer: Latest Evidence about Its Extended Use and Outcomes. Therapeutic Advances in Medical Oncology, 8, 198-208. https://doi.org/10.1177/1758834016644155

[7] Scagliotti, G.V., Parikh, P., von Pawel, J., et al. (2008) Phase III Study Comparing Cisplatin plus Gemcitabine with Cisplatin plus Pemetrexed in Chemotherapy-Naive Patients with Advanced-Stage Non-Small-Cell Lung Cancer. Journal of Clinical Oncology, 26, 3543-3551. https://doi.org/10.1200/JCO.2007.15.0375

[8] Gronberg, B.H., Bremnes, R.M., Flotten, O., et al. (2009) Phase III Study by the Norwegian Lung Cancer Study Group: Pemetrexed plus Carboplatin Compared with Gemcitabine plus Carboplatin as First-Line Chemotherapy in Advanced Non-Small Cell Lung Cancer. Journal of clinical oncology, 27, 3217-3224. https://doi.org/10.1200/JCO.2008.20.9114

[9] Ardizzoni, A., Boni, L., Tiseo, M., et al. (2007) Cisplatin- versus Carboplatin-Based Chemotherapy in First-Line Treatment of Advanced Non-Small-Cell Lung Cancer: An Individual Patient Data Meta-Analysis. Journal of the National Cancer Institute, 99, 847-857. https://doi.org/10.1093/jnci/djk196

[10] Ferry, D., Billingham, L.J, Jarrett, H.W., et al. (2011) S85 British Thoracic Oncology Group Trial, BTOG2: Randomized Phase III Clinical Trial of Gemcitabine Combined with Cisplatin 50 mg/m2 (GC50) vs. Cisplatin 80 mg/m2 (GC80) vs. Carboplatin AUC 6 (GCb6) in Advanced NSCLC. Thorax, 66, A41. https://doi.org/10.1136/thoraxjnl-2011-201054b.85

[11] Zinner, R.G., Fossella, F.V., Gladish, G.W., et al. (2005) Phase II Study of Pemetrexed in Combination with Carboplatin in the First-Line Treatment of Advanced Nonsmall Cell Lung Cancer. Cancer, 104, 2449-2456.

[12] Sequist, L.V., Yang, J.C., Yamamoto, N., et al. (2013) Phase III Study of Afatinib or Cisplatin plus Pemetrexed in Patients with Metastatic Lung Adenocarcinoma with EGFR Mutations. Journal of Clinical Oncology, 31, 3327-3334.

https://doi.org/10.1200/JCO.2012.44.2806 
Submit or recommend next manuscript to SCIRP and we will provide best service for you:

Accepting pre-submission inquiries through Email, Facebook, LinkedIn, Twitter, etc. A wide selection of journals (inclusive of 9 subjects, more than 200 journals)

Providing 24-hour high-quality service

User-friendly online submission system

Fair and swift peer-review system

Efficient typesetting and proofreading procedure

Display of the result of downloads and visits, as well as the number of cited articles Maximum dissemination of your research work

Submit your manuscript at: http://papersubmission.scirp.org/

Or contact jet@scirp.org 